\title{
Optical and Thermal Performances of (Ga,In)N/GaN Light Emitting Diodes Transferred on a Flexible Tape
}

\author{
B. Damilano, M. Lesecq, D. Zhou, E. Frayssinet, S. Chenot, J. Brault, \\ N. Defrance, A. Ebongue, Y. Cordier, and V. Hoel
}

\begin{abstract}
Blue (Ga,In)N-based light-emitting diodes (LEDs) grown on a $\mathrm{Si}(111)$ substrate by metal-organic vapor phase epitaxy are transferred on a flexible tape after the $\mathrm{Si}$ substrate removal. Their optical and thermal behaviors are measured and compared to those of regular LEDs on $\mathrm{Si}$. The light output power of the flexible LEDs is increased due to a higher light extraction efficiency related to the removal of the absorbing $\mathrm{Si}$ substrate. However, the maximum output power is limited by thermal effects due to the lower thermal conductivity of the flexible tape. Monitoring the electroluminescence wavelength of the flexible LEDs allows determining their acceptable operating range. The maximum flexible LED luminance is $5 \times 10^{5} \mathrm{~cd} / \mathrm{m}^{2}$.
\end{abstract}

Keywords - Light emitting diodes, flexible tape, GaN, InGaN.

\section{INTRODUCTION}

III-NITRIDE wide bandgap semiconductors are efficient materials for the fabrication of a variety of optoelectronic devices such as light emitting diodes [1], laser diodes [2] and of electronic devices for high-frequency and high power applications [3]. The performances of these devices are now very high. Some research efforts are currently devoted in order to get these same devices on flexible substrates as it is already the case for organic electronics [4], [5]. The expected advantage of using inorganic LEDs in place of organic LEDs is a much stronger luminance which is a key parameter for applications such as high luminance micro-displays [6], [7]. Indeed, these nitride devices are epitaxially grown on thick and rigid crystalline substrates such as sapphire or Si [8] and it is very difficult to constrain them [9]. In order to obtain flexible devices, one solution consists in transferring the nitride layer from its original rigid substrate used for epitaxy to a flexible one. This was recently demonstrated for InGaN/GaN LEDs and for AlGaN/GaN high electron mobility transistors [10]-[12]. Several strategies can be applied to transfer the LED structure. LEDs can be grown on sapphire substrate and detached using a

This work was supported in part by the technology facility network RENATECH, in part by the French National Research Agency (ANR) through the Project FLEXIGaN, and in part by the "Investissements d'Avenir" through the Program GaNeX. (Corresponding author: B. Damilano.)

B. Damilano, E. Frayssinet, S. Chenot, J. Brault, and Y. Cordier are with Université Côte d'Azur, CNRS-CRHEA, 06560 Valbonne, France (e-mail: bd@crhea.cnrs.fr).

M. Lesecq, D. Zhou, N. Defrance, and V. Hoel are with Université de Lille, CNRS, ISEN, Université de Valenciennes, UMR 8520 - IEMN, 59000 Lille, France.

A. Ebongue is with 3 M France, 95250 Beauchamp, France. laser lift-off technique [13]-[20] or can be grown on an h-BN release layer [21]. Alternatively, structures grown on silicon can be transferred after the full etching of the silicon substrate [22]-[24]. More advanced realizations such as the transfer of arrays of $\mu$ LEDs on flexible substrates have also been demonstrated [22], [25]. Most of these demonstrations are based on two-dimensional structures but 3-dimensional nanostructure such as nanowires [26], [27] or GaN microdisks [28], [29] can be used as well with the additional advantage of being stress-free and weakly affected when the flexible substrate is strongly bent. Different flexible substrates have been used such as thin metallic substrates [30], [31], plastic substrates [4]-[11], copper coated polyimide substrates [20], or ceramic substrates [32]. Metallic foils have the advantage of a good electrical and thermal conductivity but with less flexibility than plastic substrates. In this work we focus on the study of LEDs transferred on plastic substrate and especially on the assessment of their performances and their limitations related to thermal dissipation. We focus on the use of an adhesive tape with a relatively high thermal conductivity and that is electrically insulating (for plastic films). The thermal conductivity is $3 \mathrm{~W} / \mathrm{m} . \mathrm{K}$ and dielectric strength is $14.5 \mathrm{kV} / \mathrm{mm}$.

\section{EXPERIMENTAL}

The samples were grown by metal-organic chemical vapor deposition (MOCVD) on 6-inch $\mathrm{Si}(111)$ substrates in a showerhead-type Aixtron reactor with $\mathrm{H}_{2}$ or $\mathrm{N}_{2}$ carrier gases. Trimethylgallium (for $\mathrm{GaN}$ ) or triethylgallium (for $\mathrm{In}_{\mathrm{x}} \mathrm{Ga}_{1}-\mathrm{x} N$ ), trimethylindium, silane, bis(cyclopentadienyl) magnesium, and ammonia are used as precursors for gallium, indium, silicon, magnesium and nitrogen, respectively. At first, a 200-nm-thick AlN layer is deposited on the $\operatorname{Si}(111)$ substrate. Then, a stack constituted by layers of GaN (1700 nm), AlN (20 nm)/GaN $(1000 \mathrm{~nm}) / \mathrm{AlN}(20 \mathrm{~nm}) / \mathrm{GaN}(500 \mathrm{~nm})$ is grown to improve the crystalline quality and to compensate for the tensile strain coming from the thermal coefficient mismatch between the $\mathrm{GaN}$ and the $\mathrm{Si}(111)$ substrate. More details on this structure and its optimization can be found in [33]. These buffer layers are followed by $1 \mu \mathrm{m}$-thick Si:GaN n-type layer, a 5 -periods $\mathrm{In}_{0.16} \mathrm{Ga}_{0.84} \mathrm{~N}(2.4 \mathrm{~nm}) / \mathrm{GaN}(11.5 \mathrm{~nm})$ multiple quantum well active region, and then a 20-nm-thick $\mathrm{Al}_{0.2} \mathrm{Ga}_{0.8} \mathrm{~N}$ electron blocking layer and a $130 \mathrm{~nm}$ - thick GaN layer are grown, these last two layers being $\mathrm{p}$-type doped with $\mathrm{Mg}$. 


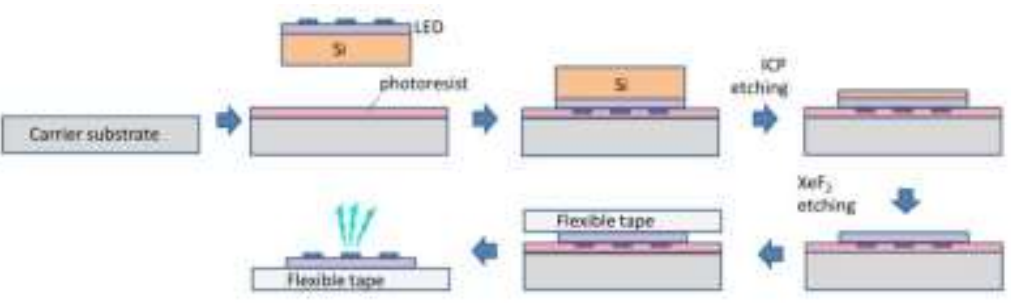

Fig. 1. Process flow for the transfer of the processed LED structure on Si substrate onto the flexible tape.
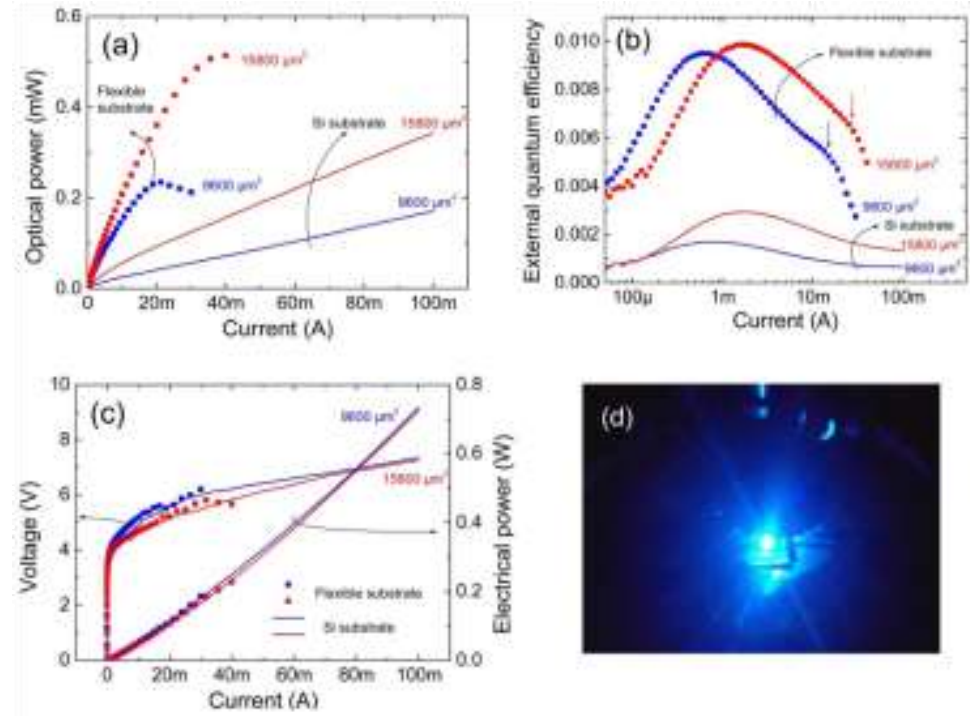

Fig. 2. Room temperature current dependence of the optical power (a), the external quantum efficiency (b), the voltage and the injected electrical power (c) of blue (Ga,In)N/GaN light emitting diodes on flexible substrates (squares) and on Si substrates (lines) for two different LED sizes: $9600 \mu \mathrm{m}^{2}$ and $15800 \mu \mathrm{m}^{2}$.

(d) Photograph of a blue LED on a flexible substrate with an area of $15800 \mu \mathrm{m}^{2}$ driven at $10 \mathrm{~mA}$.

LEDs are processed into rectangular mesas by reactive ion etching. The ohmic contacts are made of $\mathrm{Ti} / \mathrm{Al} / \mathrm{Ni} / \mathrm{Au}$ $(30 / 180 / 40 / 150 \mathrm{~nm})$ metal stacks on $\mathrm{n}-\mathrm{GaN}$ and $\mathrm{Ni} / \mathrm{Au}(5 / 5 \mathrm{~nm})$ semi-transparent current spreading layer plus a $\mathrm{Ni} / \mathrm{Au}(10 / 100$ $\mathrm{nm})$ contact pad as top electrode to $\mathrm{p}-\mathrm{GaN}$. The semitransparent area is 9600 or $15800 \mu \mathrm{m}^{2}$. The top p electrode area (contact pad) is $6000 \mu \mathrm{m}^{2}$. The LEDs are measured at room temperature under $\mathrm{CW}$ conditions. They are contacted using micro-manipulators with needle probes. The electroluminescence (EL) is detected using a

BWTek spectrometer. The LED output powers are measured with a calibrated Si photodiode located at $30 \mathrm{~mm}$ above the devices. The current-voltage characteristics are measured using a Keithley 2400 source meter.

The procedure used to transfer the LEDs onto the flexible tape is shown in Fig. 1. The tape is an experimental version based on 3M 5515 Thermal pad from 3M Company. It consists of a thermally enhanced polymer carrier coated on one side with a silicone based adhesive. The key properties were slightly improved versus the commercial version to address the particular needs of this work and to provide an enhanced thermal conductivity of $3 \mathrm{~W} / \mathrm{mK}$. The Young's modulus is 110 $\mathrm{N} / \mathrm{mm}^{2}$. The process started with the spin coating of a photoresist onto the LEDs in order to protect them. Then, the sample was flipped and temporary bonded onto a sapphire carrier substrate. Deep RIE etching and then $\mathrm{XeF}_{2}$ etching were used to gently remove the $\mathrm{Si}$ substrate without cracking the $\mathrm{GaN}$ layer. At this stage, the back side of the wafer was stuck onto the adhesive tape. Finally, the photoresist deposited on the front side was removed by immersion in acetone leading to the release of the LEDs from the carrier substrate. A similar process was previously used for the transfer of transistors based on $(\mathrm{Al}, \mathrm{Ga}) \mathrm{N} / \mathrm{GaN}$ heterostructures or $(\mathrm{Ga}, \mathrm{In}) \mathrm{N} / \mathrm{GaN}$ LEDs [10], [11], and [24].

\section{Device Characteristics}

The external quantum efficiency curves show a peak at a current (current density) of $0.7 \mathrm{~mA}\left(7 \mathrm{~A} / \mathrm{cm}^{2}\right)$ and $1.7 \mathrm{~mA}(11$ $\mathrm{A} / \mathrm{cm}^{2}$ ) for LEDs with an area of $9600 \mu \mathrm{m}^{2}$ and

$15800 \mu \mathrm{m}^{2}$, respectively. We remark that this peak efficiency is obtained almost for the same current density in LEDs on Si and in transferred LEDs. For larger injection currents, the EQE decreases. This phenomenon known as "efficiency droop" is well documented for GaN-based LEDs [34]. One of the main origins of this droop is non-radiative Auger processes which become dominant at large current densities [35]. An additional feature can be seen on the EQE curves of LEDs on flexible substrate: at a current indicated by the arrow in Fig. 2(b) ( $\sim 15 \mathrm{~mA}$ and $\sim 30 \mathrm{~mA}$ for LED sizes of $9600 \mu \mathrm{m}^{2}$ and

$\left.15800 \mu \mathrm{m}^{2}\right)$, there is an inflexion point followed by a strong decrease of the EQE. This drop in the EQE corresponds to the saturation of the output power as shown in Fig. 2(a). This phenomenon is absent for the LEDs on Si. According to the 
higher thermal conductivity of the Si substrate $(150 \mathrm{~W} / \mathrm{m} . \mathrm{K})$ compared to the flexible substrate $(3 \mathrm{~W} / \mathrm{m} . \mathrm{K})$ we can suspect that the output power saturation of the flexible LEDs is related to thermal effects that will be characterized in more details in the following. The maximum EQE (1\%) is rather low compared to the best results published by OSRAM regarding LEDs grown on Si substrate [6] and can largely be improved using optimized buffer layers and LED structures, and adding surface texturation to improve the light extraction efficiency.

The maximum LED output power of the $9600 \mu \mathrm{m}^{2}$ LED is 2 times smaller than in [25]. Our LED output power saturates at an injected electrical power of $112 \mathrm{~mW}$, i.e. 5 times larger compared to [25] due to the larger thermal conductivity of our flexible substrate. The similar LED electrical characteristics before and after transfer on the flexible substrate of Fig. 2(c) indicate that the LED transfer process does not lead to a strong change in the operating voltage. This voltage is relatively high compared to the state of the art due to non-optimized p-type doping. The injected electrical power of the LEDs on flexible substrate is very close to that of the LEDs on Si substrate. This shows that the thermal degradation observed is not linked to a strong increase of the operating voltage.

We study now the variation of the peak EL wavelength as a function of the current for LEDs with an area of $9600 \mu \mathrm{m}^{2}$. At low current, the emission wavelength $\lambda_{0}$ is respectively $480 \mathrm{~nm}$ and $479 \mathrm{~nm}$ for the LEDs on $\mathrm{Si}$ substrate and on flexible substrate. In this current range, the emission wavelength is almost constant. In the case of the LED on Si substrate, there is a blue shift of the EL peak for currents larger than $200 \mu \mathrm{A}$. At $100 \mathrm{~mA}$, the emission wavelength is $465 \mathrm{~nm}$ corresponding to a blue-shift of $15 \mathrm{~nm}$ compared to the value at low current. This blue-shift has been attributed to band filling effects and to the progressive screening of the internal electric field in the (Ga,In)N/GaN quantum wells by the injected carriers [36], [37]. In the case of the LED on flexible substrate, there is a blue-shift of the EL wavelength up to a current of $\sim 10 \mathrm{~mA}\left(104 \mathrm{~A} / \mathrm{cm}^{2}\right)$ followed by a red-shift for larger currents Fig. 3(a). This redshift is due to a strong increase of the LED temperature as shown by temperature measurement of the LED using an IR camera (Fig. 3(b)). No significant increase of the temperature can be detected for the LED on Si substrate. Therefore the wavelength emission difference ( $\lambda_{\mathrm{EL}}$ ) between the LED on flexible substrate and on Si substrate can give access to the redshift due to the temperature increase. The variation of $\lambda_{\mathrm{EL}}$ as a function of the temperature measured by the IR camera is shown in Fig. 3(c). We calculate the expected $\operatorname{In}_{0.16} \mathrm{Ga}_{0.84} \mathrm{~N}$ bandgap energy variation as a function of the temperature $\mathrm{T}$ using the Varshni expression:

$$
\Delta E(T)=-\frac{\alpha T^{2}}{\beta+T},
$$

with $\alpha=0.803 \times 10^{-3} \mathrm{eV} / \mathrm{K}^{3}$ and $\beta=797 \mathrm{~K}$. These values for the $\mathrm{In}_{0.16} \mathrm{Ga}_{0.84} \mathrm{~N}$ alloy are linearly interpolated from the data for the binary compounds $\mathrm{GaN}$ and $\mathrm{InN}$ given in [38]. This calculation gives a good agreement with the experimental data showing that the simple measurement of the EL wavelength emission of the LED as a function of the current can reliably be converted in a temperature variation.
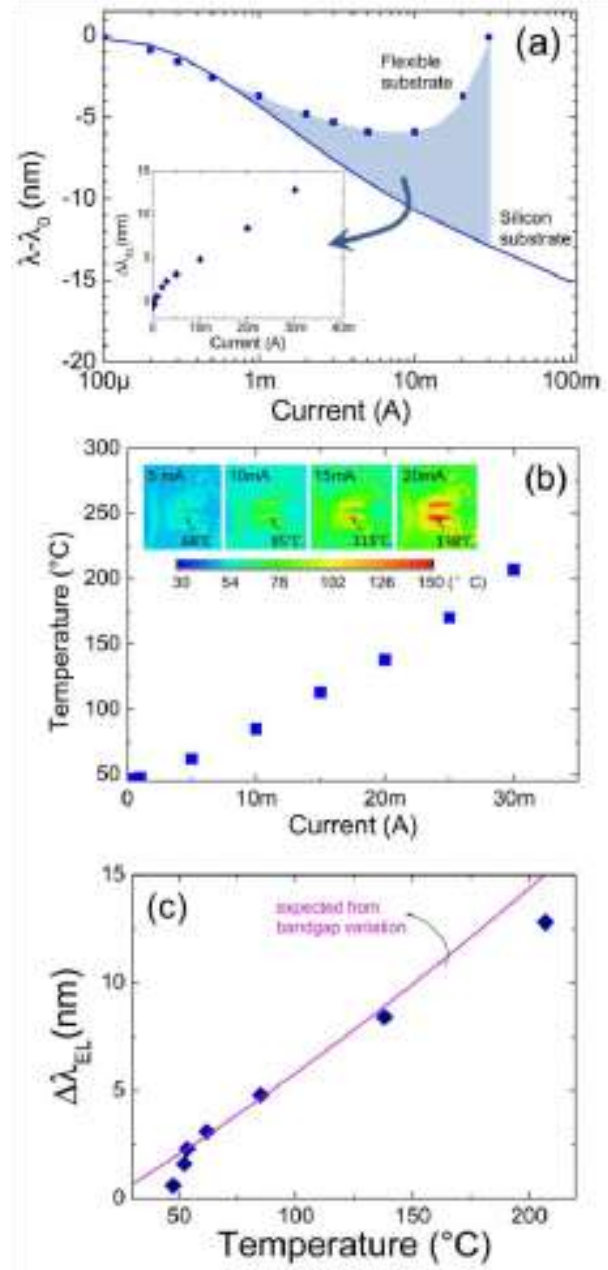

Fig. 3. (a) Electroluminescence wavelength difference $\lambda(\mathrm{I})-\lambda(\mathrm{I}<200 \mu \mathrm{A})$ as a function of the current I for light emitting diodes (LEDs) with a surface area of $9600 \mu \mathrm{m}^{2}$ on Si substrate or reported on flexible substrate. The inset shows the wavelength difference $\lambda$ EL between the two different substrates. (b) Temperature variation measured with an IR camera as a function of the current for the LED reported on flexible substrate. (c) $\lambda E L$ as a function of the temperature of the flexible LED.

We applied this technique to assess the thermal behavior of flexible LEDs during bending. To do so the sample was mounted on a half-cylinder chuck with a curvature radius of 14 $\mathrm{mm}$ (see photograph in Fig. 4). As shown in Fig. 4, the EL variations of both the bent LED and the flat LED are very similar. This shows that there is no specific change in the thermal behavior of the flexible LEDs due to the bending.

\section{CONCLUSION}

The large increase of the (Ga,In)N/GaN LED temperature during operation at large current limits the maximum output power which can be achieved with these flexible LEDs. However, we can point out that if lower light levels are acceptable, the flexible LEDs can be driven at the current corresponding to the maximum external quantum efficiency. 
In this case no strong thermal effects are present. For the LED with an area of $15800 \mu \mathrm{m}^{2}$, at a current of $1.7 \mathrm{~mA}$, an output power of $46 \mu \mathrm{W}$ is obtained and a luminance of

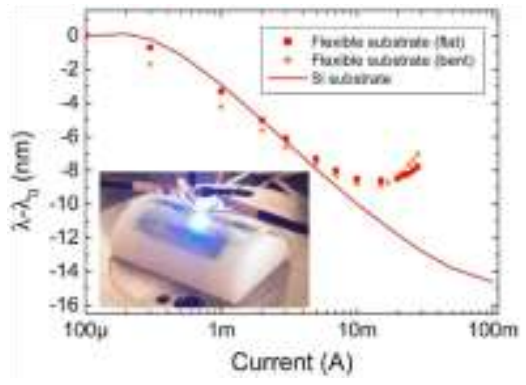

Fig. 4. Electroluminescence wavelength difference $\lambda(\mathrm{I})-\lambda(\mathrm{I}<200 \mu \mathrm{A})$ as a function of the current I for light emitting diodes with an area of $15800 \mu \mathrm{m}^{2}$ on $\mathrm{Si}$ substrate or reported on flexible substrate. The flexible light emitting diode is measured under flat conditions or bent conditions with a curvature radius of $14 \mathrm{~mm}$.

$4 \times 10^{4} \mathrm{~cd} / \mathrm{cm}^{2}$ can be deduced (considering an isotropic emission). The maximum luminance is $5 \times 10^{5} \mathrm{~cd} / \mathrm{cm}^{2}$ at a

current of $40 \mathrm{~mA}$. Even with a strong limitation of the injected current, the luminance that we obtained favorably compares with the one achievable in organic LEDs. If larger output powers are required, the LED series resistance has to be minimized to decrease the Joule heating for a given current.

\section{ACKNOWLEDGMENT}

The authors would like to thank M. Leroux, J. Y. Duboz, P. De Mierry, and J. Massies for the critical reading of the manuscript.

\section{REFERENCES}

[1] S. Nakamura, T. Mukai, and M. Senoh, "Candela-class high-brightness InGaN/AlGaN double-heterostructure blue-light-emitting diodes," Appl. Phys. Lett., vol. 64, no. 13, pp. 1687-1689, Mar. 1994.

[2] S. Nakamura et al., "Room-temperature continuous-wave operation of InGaN multi-quantum-well structure laser diodes," Appl. Phys. Lett., vol. 69, no. 26, pp. 4056-4058, Dec. 1996.

[3] T. Palacios et al., "High-power AlGaN/GaN HEMTs for Ka-band applications," IEEE Electron Device Lett., vol. 26, no. 11, pp. 781-783, Nov. 2005.

[4] T. Sekitani, U. Zschieschang, H. Klauk, and T. Someya, "Flexible organic transistors and circuits with extreme bending stability," Nature Mater. vol. 9, pp. 1015-1022, Nov. 2010.

[5] Z. B. Wang et al., "Unlocking the full potential of organic light-emitting diodes on flexible plastic," Nature Photon., vol. 5, no. 12, pp. 753-757, Oct. 2011

[6] Z. Y. Fan, J. Y. Lin, and H. X. Jiang, "III-nitride micro-emitter arrays: Development and applications," J. Phys. D, Appl. Phys., vol. 41, no. 9, p. 094001, May 2008.

[7] F. Templier, "GaN-based emissive microdisplays: A very promising technology for compact, ultra-high brightness display systems," J. Soc. Inf. Display, vol. 24, no. 11, pp. 669-675, Nov. 2016.

[8] B. Hahn, B. Galler, and K. Engl, "Development of high-efficiency and high-power vertical light emitting diodes," Jpn. J. Appl. Phys., vol. 53, no. 10 , p. 100208 , Oct. 2014.

[9] J. Xu et al., "Effects of polarization-field tuning in GaInN light-emitting diodes," Appl. Phys. Lett., vol. 99, no. 4, p. 041105, Jul. 2011.

[10] M. Lesecq, V. Hoel, A. L. des Etangs-Levallois, E. Pichonat, Y. Douvey, and J. C. De Jaeger, "High performance of AlGaN/GaN HEMTs reported on adhesive flexible tape," IEEE Electron Device Lett., vol. 32, no. 2, pp. 143-145, Feb. 2011.

[11] N. Defrance et al., "Fabrication, characterization, and physical analysis of AlGaN/GaN HEMTs on flexible substrates," IEEE Trans. Electron Devices, vol. 60, no. 3, pp. 1054-1059, Mar. 2013.

[12] S. K. Oh et al., "High-power flexible $\mathrm{AlGaN} / \mathrm{GaN}$ heterostructure fieldeffect transistors with suppression of negative differential conductance," Appl. Phys. Lett., vol. 111, no. 13, p. 133502, Sep. 2017.

[13] Y. Jung et al., "GaN-based light-emitting diodes on origami substrates," Appl. Phys. Lett., vol. 100, no. 23, p. 231113, Jun. 2012.

[14] J. Chun et al., "Transfer of GaN LEDs from sapphire to flexible substrates by laser lift-off and contact printing," IEEE Photon. Technol. Lett., vol. 24, no. 23, pp. 2115-2118, Dec. 1, 2012.

[15] T. Kim et al., "Temperature- and size-dependent characteristics in ultrathin inorganic light-emitting diodes assembled by transfer printing," Appl. Phys. Lett., vol. 104, no. 5, p. 051901, Feb. 2014.

[16] W.-S. Choi, H. J. Park, S.-H. Park, and T. Jeong, "Flexible InGaN LEDs on a polyimide substrate fabricated using a simple direct-transfer method," IEEE Photon. Technol. Lett., vol. 26, no. 21, pp. 2115-2117, Nov. 1, 2014.

[17] W.-S. Choi et al., "Characterization of flexible InGaN LEDs with various curvatures," Mater. Lett., vol. 165, pp. 252-256, Feb. 2016.

[18] R.-H. Horng, C.-H. Tien, S.-H. Chuang, K.-C. Liu, and D.-S. Wuu, "External stress effects on the optical and electrical properties of flexible InGaN-based green light-emitting diodes," Opt. Express, vol. 23, no. 24, pp. 31334-31341, Nov. 2015.

[19] J. H. Seo et al., "A simplified method of making flexible blue LEDs on a plastic substrate," IEEE Photon. J., vol. 7, no. 2, Apr. 2015, Art. no. 8200207.

[20] L. Li et al., "Heterogeneous integration of microscale GaN lightemitting diodes and their electrical, optical, and thermal characteristics on flexible substrates," Adv. Mater. Technol., vol. 3, no. 1, p. 1700239, Jan. 2018.

[21] T. Makimoto, K. Kumakura, Y. Kobayashi, T. Akasaka, and H. Yamamoto, "A vertical InGaN/GaN light-emitting diode fabricated on a flexible substrate by a mechanical transfer method using BN," Appl. Phys. Express, vol. 5, no. 7, p. 072102, Jun. 2012.

[22] H.-S. Kim et al., "Unusual strategies for using indium gallium nitride grown on silicon (111) for solid-state lighting," Proc. Nat. Acad. Sci. USA, vol. 108, no. 25, pp. 10072-10077, 2011.

[23] X. Zou, X. Zhang, W. C. Chong, C. W. Tang, and K. M. Lau, "Vertical LEDs on rigid and flexible substrates using GaN-on-Si epilayers and Aufree bonding," IEEE Trans. Electron Devices, vol. 63, no. 4, pp. 15871593, Apr. 2016.

[24] G. Tabares et al., "Impact of the bending on the electroluminescence of flexible InGaN/GaN light-emitting diodes," IEEE Photon. Technol. Lett., vol. 28, no. 15, pp. 1661-1664, Aug. 1, 2016.

[25] T. Kim et al., "High-efficiency, microscale GaN light-emitting diodes and their thermal properties on unusual substrates," Small, vol. 8, no. 11, pp. 1643-1649, Jun. 2012.

[26] K. Chung et al., "Growth and characterizations of GaN micro-rods on graphene films for flexible light emitting diodes," APL Mater., vol. 2, no. 9, p. 092512, Sep. 2014.

[27] X. Dai et al., "Flexible light-emitting diodes based on vertical nitride nanowires," Nano Lett., vol. 15, no. 10, pp. 6958-6964, Oct. 2015.

[28] J. H. Choi et al., "Fully flexible GaN light-emitting diodes through nanovoid-mediated transfer," Adv. Opt. Mater., vol. 2, no. 3, pp. 267274, Mar. 2014.

[29] K. Chung et al., "Flexible GaN light-emitting diodes using GaN microdisks epitaxial laterally overgrown on graphene dots," Adv. Mater., vol. 28, no. 35, pp. 7688-7694, Sep. 2016.

[30] P. Tian et al., "Fabrication, characterization and applications of flexible vertical InGaN micro-light emitting diode arrays," Opt. Express, vol. 24, no. 1, pp. 699-707, Jan. 2016.

[31] H. Kim et al., "Fabrication of full-color GaN-based light-emitting diodes on nearly lattice-matched flexible metal foils," Sci. Rep., vol. 7, Dec. 2017, Art. no. 2112

[32] S. H. Kim et al., "Visible flip-chip light-emitting diodes on flexible ceramic substrate with improved thermal management," IEEE Electron Device Lett., vol. 37, no. 5, pp. 615-617, May 2016.

[33] E. Frayssinet, Y. Cordier, H. P. D. Schenk, and A. Bavard, "Growth of thick GaN layers on 4-in. and 6-in. silicon (111) by metal-organic vapor phase epitaxy," Phys. Status Solidi C, vol. 8, no. 5, pp. 1479-1482, May 2011. 
[34] M.-H. Kim et al., "Origin of efficiency droop in GaN-based lightemitting diodes," Appl. Phys. Lett., vol. 91, no. 18, p. 183507, 2007.

[35] Y. C. Shen, G. O. Mueller, S. Watanabe, N. F. Gardner, A. Munkholm, and M. R. Krames, "Auger recombination in InGaN measured by photoluminescence," Appl. Phys. Lett., vol. 91, no. 14, p. 141101, 2007.

[36] T. Mukai, K. Takekawa, and S. Nakamura, "InGaN-based blue lightemitting diodes grown on epitaxially laterally overgrown $\mathrm{GaN}$ substrates," Jpn. J. Appl. Phys., vol. 37, no. 7B, pp. L839-L841, Jul. 1998.

[37] F. D. Sala et al., "Free-carrier screening of polarization fields in wurtzite GaN/InGaN laser structures," Appl. Phys. Lett., vol. 74, no. 14, pp. 2002 2004, Apr. 1999.

[38] I. Vurgaftman and J. R. Meyer, "Band parameters for nitrogen-containing semiconductors," J. Appl. Phys., vol. 94, no. 6, pp. 3675-3696, 2003. 